\title{
Meromorphic close-to-convex functions satisfying a differential inequality
}

Kuldeep Kaur Shergill and Sukhwinder Singh Billing

Abstract. In the present paper, we study the differential inequality

$$
-\Re\left[(1-\alpha) z^{2} f^{\prime}(z)+\alpha\left(1+\frac{z f^{\prime \prime}(z)}{f^{\prime}(z)}\right)\right]>\beta,(z \in \mathbb{E})
$$

where $f \in \Sigma$ and notice that the members of class $\Sigma$ which satisfy the above inequality are meromorphic close-to-convex.

Mathematics Subject Classification (2010): 30C45, 30C80.

Keywords: Meromorphic function, meromorphic starlike function, meromorphic close-to-convex function.

\section{Introduction}

Let $\Sigma$ denote the class of meromorphic functions of the form

$$
f(z)=\frac{1}{z}+\sum_{n=1}^{\infty} a_{n} z^{n},
$$

which are analytic in the punctured open unit disc $\mathbb{E}_{0}=\mathbb{E} \backslash\{0\}$, where

$$
\mathbb{E}=\{z \in \mathbb{C}:|z|<1\} .
$$

A function $f \in \Sigma$ is said to be meromorphic starlike of order $\alpha$ if and only if

$$
-\Re\left(\frac{z f^{\prime}(z)}{f(z)}\right)>\alpha,(z \in \mathbb{E})
$$

for some real $\alpha(0 \leq \alpha<1)$. The class of such functions is denoted by $\mathcal{M S}^{*}(\alpha)$. Write $\mathcal{M S}^{*}=\mathcal{M S}^{*}(0)$, the class of meromorphic starlike functions i.e. meromorphic functions which satisfy the condition

$$
-\Re\left(\frac{z f^{\prime}(z)}{f(z)}\right)>0,(z \in \mathbb{E}) .
$$


A function $f \in \Sigma$ is said to be meromorphic close-to-convex of order $\alpha$ if there exists a meromorphic starlike function $g \in \mathcal{M S}^{*}$ such that

$$
-\Re\left(\frac{z f^{\prime}(z)}{g(z)}\right)>\alpha,(z \in \mathbb{E}) .
$$

The class of such functions is denoted by $\mathcal{M C}(\alpha)$. Write $\mathcal{M C}=\mathcal{M C}(0)$, the class of meromorphic close-to-convex functions i.e. meromorphic functions which satisfy the condition

$$
-\Re\left(\frac{z f^{\prime}(z)}{g(z)}\right)>0,(z \in \mathbb{E})
$$

where $g \in \mathcal{M S}^{*}$.

A little calculation yields that the function $g(z)=\frac{1}{z}$ is a member of class $\mathcal{M S}^{*}$. Therefore, the condition (1.1) reduces to the following condition

$$
-\Re\left(z^{2} f^{\prime}(z)\right)>0,(z \in \mathbb{E}) .
$$

Therefore, $f \in \mathcal{M C}$ if $-\Re\left(z^{2} f^{\prime}(z)\right)>0$.

In the literature of meromorphic functions, many authors obtained the conditions for meromorphic close-to-convex functions. Some of the results from literature are given below:

Jing and $\mathrm{Li}[4]$ have proved the following results:

Theorem 1.1. For any $f \in \Sigma$, suppose that for arbitrary $\alpha, f$ satisfies $-z^{2} f^{\prime}(z) \neq \alpha$ and the following inequalities:

(i) For the case $0<\alpha<\frac{1}{2}$

$$
2+\Re\left(\frac{z f^{\prime \prime}(z)}{f^{\prime}(z)}\right)<\frac{\alpha}{2(1-\alpha)},
$$

(ii) For the case $\frac{1}{2} \leq \alpha<1$

$$
2+\Re\left(\frac{z f^{\prime \prime}(z)}{f^{\prime}(z)}\right)<\frac{1-\alpha}{2 \alpha},
$$

then $f \in \mathcal{M C}(\alpha)$.

Theorem 1.2. Let $f \in \Sigma$, suppose that for arbitrary $\alpha, f$ satisfies $-z^{2} f^{\prime}(z) \neq \alpha$ and the following inequality:

$$
1+\Re\left(\frac{z f^{\prime \prime}(z)}{f^{\prime}(z)}\right) \geq \frac{3 \alpha-2}{2(1-\alpha)}
$$

then $f \in \mathcal{M C}(\alpha)$.

Goyal and Prajapat [1] proved the following results:

Theorem 1.3. If $f \in \Sigma$ satisfies the following inequality

$$
\left|\frac{z f^{\prime \prime}(z)}{f^{\prime}(z)}-z^{2} f^{\prime}(z)+1\right|<\frac{(1-\alpha)(3-\alpha)}{2-\alpha} \quad(0 \leq \alpha<1),
$$

then $f \in \mathcal{M C}(\alpha)$. 
Theorem 1.4. If $f \in \Sigma$ satisfies the following inequality

$$
\left|\frac{z f^{\prime \prime}(z)}{f^{\prime}(z)}-z^{2} f^{\prime}(z)+1\right|<\frac{3}{2}
$$

then $f \in \mathcal{M} C$.

Theorem 1.5. If $f \in \Sigma$ satisfies the following inequality

then $f \in \mathcal{M C}$.

$$
\Re\left[z^{2}\left\{f^{\prime}(z)\left(z^{2} f^{\prime}(z)-1\right)-z f^{\prime \prime}(z)\right\}\right]>-\frac{1}{2},
$$

Recently Wang and Guo [3] proved the following results:

Theorem 1.6. Let $f \in \Sigma$ and suppose that there exists a meromorphic starlike function $g$ such that

$$
\Re\left\{\frac{z f^{\prime}(z)}{g(z)}\left(1+\frac{z f^{\prime \prime}(z)}{f^{\prime}(z)}-\frac{z g^{\prime}(z)}{g(z)}\right)\right\}>\frac{1}{2}\left(1+\left|\frac{z f^{\prime}(z)}{g(z)}\right|^{2}\right),
$$

then $f \in \mathcal{M} C$.

Theorem 1.7. Let $f \in \Sigma$ and suppose that there exists a meromorphic starlike function g such that

$$
\Re\left\{\frac{z f^{\prime}(z)}{g(z)}\left(-1-\frac{z f^{\prime \prime}(z)}{f^{\prime}(z)}+\frac{z g^{\prime}(z)}{g(z)}\right)\right\}>-\frac{1}{4}\left(1+\left|\frac{z f^{\prime}(z)}{g(z)}\right|^{2}\right),
$$

then $f \in \mathcal{M} C\left(\frac{1}{2}\right)$.

Theorem 1.8. For $f \in \Sigma$, suppose that there exists a meromorphic starlike function $g$ such that

$$
\Re\left\{\frac{z f^{\prime}(z)}{g(z)}\left(-1-\frac{z f^{\prime \prime}(z)}{f^{\prime}(z)}+\frac{z g^{\prime}(z)}{g(z)}\right)\right\}>-\frac{1}{2}(1-\alpha),(0 \leq \alpha<1)
$$

then $f \in \mathcal{M C}(\alpha)$.

\section{Preliminaries}

We shall need the following lemma of Miller and Mocanu [2] to prove our main result.

Lemma 2.1. Let $\mathbb{D}$ be a subset of $\mathbb{C} \times \mathbb{C}(\mathbb{C}$ is the complex plane) and let $\phi: \mathbb{D} \rightarrow \mathbb{C}$ be a complex function. For $u=u_{1}+i u_{2}, v=v_{1}+i v_{2}\left(u_{1}, u_{2}, v_{1}, v_{2}\right.$ are reals), let $\phi$ satisfy the following conditions:

(i) $\phi(u, v)$ is continuous in $\mathbb{D}$;

(ii) $(1,0) \in \mathbb{D}$ and $\Re \phi(1,0)>0$; and

(iii) $\Re\left\{\phi\left(i u_{2}, v_{1}\right)\right\} \leq 0$ for all $\left(i u_{2}, v_{1}\right) \in \mathbb{D}$ such that $v_{1} \leq-\left(1+u_{2}^{2}\right) / 2$.

Let $p(z)=1+p_{1} z+p_{2} z^{2}+\ldots$ be regular in the unit disc $\mathbb{E}$ such that $\left(p(z), z p^{\prime}(z)\right) \in \mathbb{D}$ for all $z \in \mathbb{E}$. If

$$
\Re\left[\phi\left(p(z), z p^{\prime}(z)\right)\right]>0, z \in \mathbb{E},
$$

then $\Re p(z)>0, z \in \mathbb{E}$. 


\section{Main theorem}

Theorem 3.1. Let $\alpha$ and $\beta$ be real numbers such that $\alpha \leq \beta<1$. If $f \in \Sigma$ satisfies

$$
-\Re\left[(1-\alpha) z^{2} f^{\prime}(z)+\alpha\left(1+\frac{z f^{\prime \prime}(z)}{f^{\prime}(z)}\right)\right]>\beta, z \in \mathbb{E},
$$

then $-\Re\left(z^{2} f^{\prime}(z)\right)>0$ in $\mathbb{E}$. So, $f$ is meromorphic close-to-convex in $\mathbb{E}$. The result is sharp in the sense that the constant $\beta$ on the right hand side of (3.1) cannot be replaced by a real number smaller than $\alpha$.

Proof. Define a function $p$ by $p(z)=-z^{2} f^{\prime}(z)$ where $p$ is analytic in $\mathbb{E}$. Then,

$$
\begin{aligned}
& -\left[(1-\alpha) z^{2} f^{\prime}(z)+\alpha\left(1+\frac{z f^{\prime \prime}(z)}{f^{\prime}(z)}\right)\right] \\
= & -\left[(1-\alpha)(-p(z))+\alpha\left(-1+\frac{z p^{\prime}(z)}{p(z)}\right)\right]
\end{aligned}
$$

Thus, condition (3.1) is equivalent to

$$
\Re\left[\frac{1-\alpha}{1-\beta} p(z)-\frac{\alpha}{1-\beta} \frac{z p^{\prime}(z)}{p(z)}+\frac{\alpha-\beta}{1-\beta}\right]>0, z \in \mathbb{E} .
$$

If $\mathbb{D}=(\mathbb{C} \backslash\{0\}) \times \mathbb{C}$, define $\phi(u, v): \mathbb{D} \rightarrow \mathbb{C}$ as under:

$$
\phi(u, v)=\frac{1-\alpha}{1-\beta} u-\frac{\alpha}{1-\beta} \frac{v}{u}+\frac{\alpha-\beta}{1-\beta} .
$$

Then $\phi(u, v)$ is continuous in $\mathbb{D},(1,0) \in D$ and $\Re(\phi(1,0))=1>0$. Further, in view of $(3.3)$,

$$
\Re\left[\phi\left(p(z), z p^{\prime}(z)\right)\right]>0, z \in \mathbb{E} .
$$

Let $u=u_{1}+i u_{2}, v=v_{1}+i v_{2}\left(u_{1}, u_{2}, v_{1}, v_{2}\right.$ are real numbers). Then, for $\left(i u_{2}, v_{1}\right) \in \mathbb{D}$, with $v_{1} \leq-\frac{1+u_{2}^{2}}{2}$, we have

$$
\Re\left[\phi\left(i u_{2}, v_{1}\right)\right]=\Re\left[\frac{1-\alpha}{1-\beta} i u_{2}-\frac{\alpha}{1-\beta} \frac{v_{1}}{i u_{2}}+\frac{\alpha-\beta}{1-\beta}\right]=\frac{\alpha-\beta}{1-\beta} \leq 0 .
$$

In view of Lemma 2.1, proof now follows.

To show that the constant $\beta$ on the right side of (3.1) cannot be replaced by a real number smaller than $\alpha$, we consider the function

$$
f_{0}(z)=\frac{-z-2 \log (1-z)}{z^{2}}
$$

which belongs to the class $\Sigma$. A simple calculation gives

$$
\begin{aligned}
-\left[(1-\alpha) z^{2} f_{0}^{\prime}(z)+\alpha(1\right. & \left.\left.+\frac{z f_{0}^{\prime \prime}(z)}{f_{0}^{\prime}(z)}\right)\right] \\
= & -(1-\alpha)\left[\frac{-z^{2}+3 z+4(1-z) \log (1-z)}{z(1-z)}\right] \\
& -\alpha\left[\frac{-z^{3}+10 z^{2}-7 z-8(1-z)^{2} \log (1-z)}{z^{3}-4 z^{2}+3 z+4(1-z)^{2} \log (1-z)}\right]
\end{aligned}
$$


Using Mathematica 7.0, we plot in Figure 3.1, the image of the unit disc $\mathbb{E}$ under the operator

$$
-\left[(1-\alpha) z^{2} f_{0}^{\prime}(z)+\alpha\left(1+\frac{z f_{0}^{\prime \prime}(z)}{f_{0}^{\prime}(z)}\right)\right]
$$

taking $\alpha=-1$.

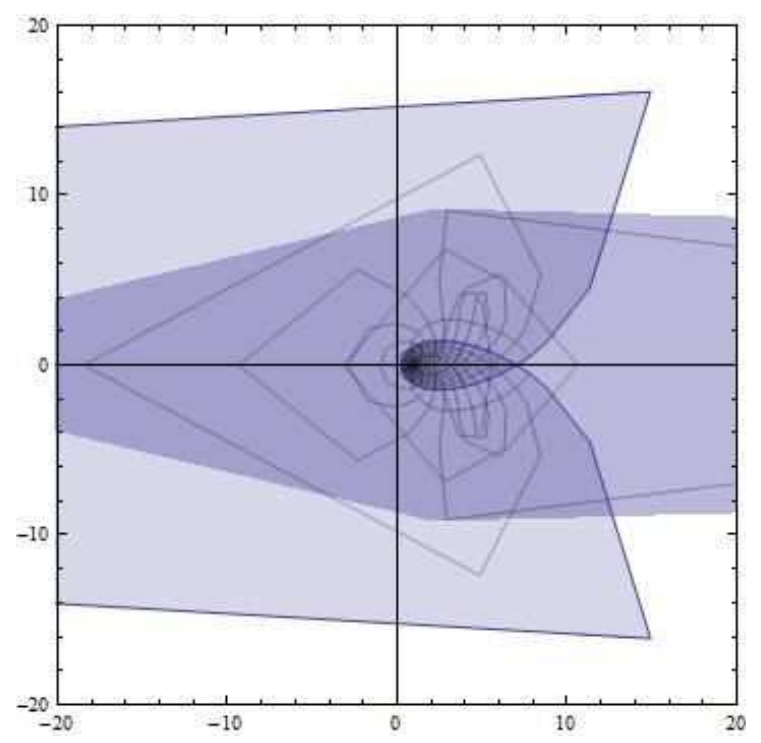

Figure 3.1

From Figure 3.1, we observe that minimum real part of

$$
-\left[(1-\alpha) z^{2} f_{0}^{\prime}(z)+\alpha\left(1+\frac{z f_{0}^{\prime \prime}(z)}{f_{0}^{\prime}(z)}\right)\right] \text { for } \alpha=-1
$$

is smaller than -1 (the chosen value of $\alpha$ ).

In Figure 3.2, we plot the image of unit disc $\mathbb{E}$ under the function $-z^{2} f_{0}^{\prime}(z)$.

It is obvious that $-\Re\left(z^{2} f_{0}^{\prime}(z)\right) \ngtr 0$ for all $\mathrm{z}$ in $\mathbb{E}$.

Moreover, the point $z=0.9$ is an interior point of $\mathbb{E}$, but at this point

$$
-\Re\left(z^{2} f_{0}^{\prime}(z)\right)=-10.766 \ldots<0 .
$$




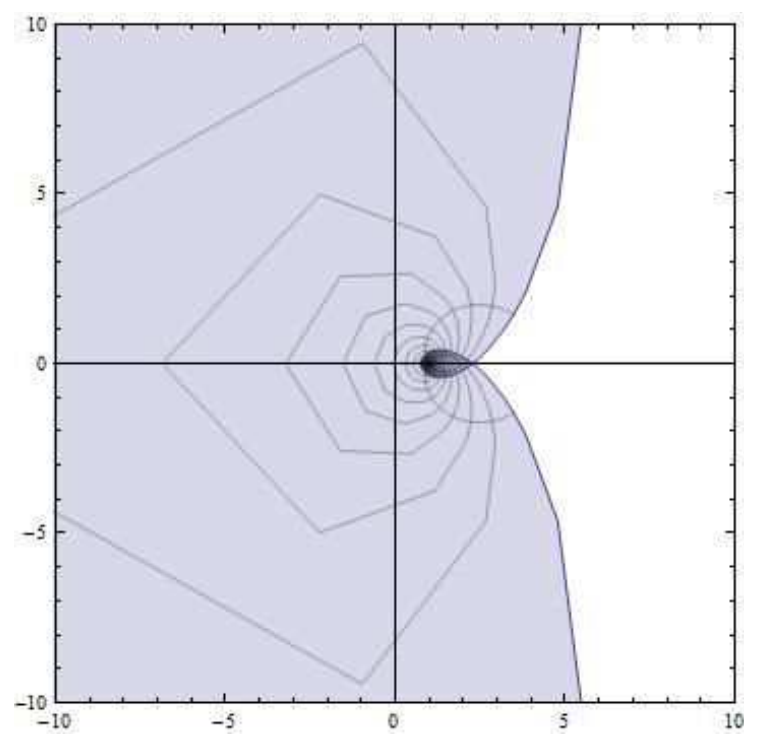

Figure 3.2

This justifies our claim.

\section{References}

[1] Goyal, S.P., Prajapat J.K., A new class of meromorphic functions involving certain linear operator, Tamsui Oxford J. Math. Sci., (25)(2009), no. 2, 167-176.

[2] Miller, S.S., Mocanu, P.T., Differential subordinations and inequalities in the complex plane, J. Differential Equations, (67)(1987), 199-211.

[3] Wang Jing, Guo Lifeng, Sufficient conditions for meromorphic close-to-convex functions, Int. Electron. J. Pure Appl. Math., (3)(2014), 375-379.

[4] Wang Jing, Li Bo, Sufficient conditions for meromorphic close-to-convexity of order $\alpha$, Sch. J. Eng. Tech., (2)(2B)(2014), 305-308.

Kuldeep Kaur Shergill

Sri Guru Granth Sahib World University,

Department of Mathematics,

Fatehgarh Sahib-140407 (Punjab), India

e-mail: kkshergill16@gmail.com

Sukhwinder Singh Billing

Sri Guru Granth Sahib World University,

Department of Mathematics,

Fatehgarh Sahib-140407 (Punjab), India

e-mail: ssbilling@gmail.com 